\title{
Evaluation of the Twofold Gaussian Mixture Model applied to Clinical Volume Datasets
}

\author{
Jan Bruijns \\ Philips Research Eindhoven, The Netherlands \\ jan.bruijns@philips.com
}

\begin{abstract}
Volume representations of blood vessels acquired by 3D rotational angiography are very suitable for diagnosing a stenosis or an aneurysm. For optimal treatment, physicians need to know the shape of the diseased vessel parts. Binary segmentation by thresholding is the first step in our shape extraction procedure. Assuming a twofold Gaussian mixture model (GMM), the model parameters (and thus the threshold for binary segmentation) can be extracted by the Expectation-Maximization (EM) algorithm. The question is whether this GMM threshold gives a good segmentation. Therefore we compared segmentations induced by the GMM threshold with segmentations induced by thresholds derived in a different way. It appeared that a twofold Gaussian mixture model is not always a correct assumption for the distribution of the gray values.
\end{abstract}

\section{Introduction}

Volume representations of blood vessels acquired by 3D rotational angiography after injection with a contrast agent [1] have a clear distinction in gray values between tissue and vessel voxels. Therefore, these volume representations are very suitable for diagnosing a stenosis, a local narrowing of a vessel caused for example by cholesterol, or an aneurysm, a local widening of a vessel caused by a weak vessel wall.

For optimal treatment of a stenosis or an aneurysm, physicians need to know the cross-sectional shape parameters in the neighborhood of the diseased vessel parts. The starting point for many shape extraction methods is a segmented volume. Such a segmented volume can be created by thresholding (given further detail in Section 2.3).

To eliminate inter- and intra-operator variations this threshold should be extracted automatically from the data. Starting point for automatic threshold extraction can be a twofold Gaussian mixture model $[2,3,4]$. The question is whether the threshold derived from such a model results in a good segmentation. If the threshold is too low, many noise bulges arise; if the threshold is too high, an undersized vessel surface results.

\subsection{Related work}

Gan et al. introduced a method for vascular segmentation of 3D rotational angiography volumes based on MIP images [3]. Applying the Expectation- 
Maximization (EM) algorithm for a twofold Gaussian mixture model, they estimate a threshold from a MIP image because the standard deviation of the individual classes in a MIP image is smaller. This threshold is used to classify the voxels belonging to this MIP image (i.e. the voxels with the maximum gray value in the projection direction). After the resulting vessel voxels are labelled in a corresponding binary volume, their gray values in the original volume is set to zero, and the procedure is repeated until over fitting of the non-vessel class is detected. This iterative algorithm is applied in the three axis directions and the resulting segmentations are combined by minimizing an energy functional for a spatially smooth result.

\subsection{What is new}

Since segmentations of our clinical volume datasets by one or more experienced radiologist were not available, we developed a new method to quantify segmentations on the basis of the gradient information of the gray value volumes (given further detail in Section 2.4).

Since we apply this method to quantify not only segmentations induced by the GMM threshold but also segmentations induced by thresholds derived in a different way (given further detail in Section 2.2), we can assess the segmentations and thus the GMM threshold.

\section{Method}

\subsection{EM algorithm}

Since the Expectation-Maximization (EM) algorithm [5, 6] requires a number of iterations through the observations (e.g. the gray values), and because of the large number of observations (e.g. $512 \times 512 \times 512$ volumes), the EM algorithm is very time-consuming. To accelerate the extraction of the parameters of a twofold Gaussian mixture model (GMM) by the EM algorithm, we use the variant developed by Bruijns [4]. He applies the EM algorithm on to the histogram of the observations, requiring a single pass through the volume and a number of iterations through the much smaller histogram. After the parameters of the Gaussian mixture model are extracted, the GMM threshold is given by the intersection point of the two Gaussian components.

\subsection{Bench mark thresholds}

As already told in Section 1.2, we have not only used the GMM threshold for binary segmentation but also thresholds extracted in a different way from the gray value volumes. We have used the threshold proposed in [7] (indicated by "OT"), the threshold proposed in [2] (indicated by "KI"), the threshold proposed in [8] (indicated by "RF") and the threshold proposed in [9] (indicated by "GR"). 


\subsection{Segmentation}

As explained in Section 2.1 of [9], binary segmentation of a gray value volume with a single threshold gives too much noise. To remove this noise, the gray value volume is transformed to a segmented volume by a region growing algorithm with two thresholds [10].

Voxels with a gray value below the lower threshold are classified as tissue voxels. Voxels with a gray value above the upper threshold are classified as vessel voxels. Voxels with a gray value between the two thresholds are classified as potential vessel voxels. A potential vessel voxel is classified as vessel voxel if it is face connected to a vessel voxel, possibly via a chain of face connected potential vessel voxels.

The GMM threshold and the bench mark thresholds (Sec. 2.2) are used as lower threshold. The upper thresholds are derived from the lower thresholds and the gray value volume similar as in Section 2.2 of [9].

\subsection{Evaluation}

The true boundary between vessels and tissue is located at (a subset of) the positions $\left\{\mathbf{p}_{\max }\right\}$ of the maxima of the gradient magnitude $\|\mathbf{g}(\mathbf{p})\|$ in the direction of the gradient vector $\mathbf{n}(\mathbf{p})$. These positions are given by the following relation:

$$
\left\|\mathbf{g}\left(\mathbf{p}_{\max }+\mathbf{n}\left(\mathbf{p}_{\max }\right) * d t\right)\right\|<\left\|\mathbf{g}\left(\mathbf{p}_{\max }\right)\right\|
$$

Since the high gray value gradient ridge voxels (Section 2.3.2 of [9]) are located close to the maxima of the gradient magnitude, these voxels are used as reference boundary for a quantitative evaluation of the segmentations.

Since the Manhattan distance transform [11] with regard to the high gray value gradient ridge voxels gives the Manhattan distance between every voxel and its closest high gray value ridge voxel, and since the Manhattan distance transform is faster to compute than the Euclidian distance transform, we have used these Manhattan distances to indicate the segmentation performance. We have computed per volume the following characteristic numbers from the Manhattan distances of the border vessel voxels (i.e. vessel voxels with a tissue voxel as neighbor):

1. The fraction of border vessel voxels with a Manhattan distance greater than zero (i.e. the border vessel voxels not located on the high gray value ridges). This fraction is indicated by "border_off_ridges".

2. The average Manhattan distance of the border vessel voxels (indicated by "avg_dist2ridges").

3. The maximum Manhattan distance of the border vessel voxels (indicated by "max_dist2ridges"). 
Table 1. Median $M D$, mean $M N$ and maximum $M X$ of the three characteristic numbers.

\begin{tabular}{lrrrrrrrrr}
\hline & \multicolumn{3}{c}{ "border_on_ridges" } & \multicolumn{3}{c}{ "avg_dist2ridges" } & \multicolumn{3}{c}{ "max_dist2ridges" } \\
& $M D$ & $M N$ & $M X$ & $M D$ & $M N$ & $M X$ & $M D$ & $M N$ & $M X$ \\
\hline GMM & 0.3839 & 0.4253 & 0.9854 & 0.4345 & 1.0924 & 20.9937 & 7 & 12.1938 & 93 \\
OT & 0.3394 & 0.3522 & 0.8499 & 0.3516 & 0.6760 & 19.6738 & 4 & 7.3718 & 91 \\
KI & 0.3692 & 0.3887 & 0.8875 & 0.4022 & 0.4501 & 1.8899 & 6 & 8.1125 & 35 \\
RF & 0.3770 & 0.3988 & 0.9352 & 0.4016 & 0.4738 & 2.1754 & 6 & 9.6296 & 46 \\
GR & 0.3176 & 0.3274 & 0.6537 & 0.3479 & 0.3707 & 1.7681 & 4 & 5.5988 & 33 \\
\hline
\end{tabular}

\subsection{Remarks}

1. When the gradient ridges are used for extraction of the lower threshold (Section 2.3.3 of [9]), faultless detecting of all gradient ridge voxels at all vessel-tissue boundaries is not required. Better a number of gradient ridge voxels at the vessel-tissue boundaries ruled out than a number of voxels at possible artefacts included. But, when the gradient ridges are used for quantitative evaluation of segmentations, it is better that a number of voxels at possible artefacts are included, than that a number of gradient ridge voxels at the vessel-tissue boundaries are ruled out. Indeed, a significant loss of gradient ridge voxels at the vessel-tissue boundaries result in overestimated characteristic numbers.

2. In case of a "perfect segmentation" the vessel boundary is coincident with the high gray value gradient ridges. In this case all border vessel voxels are located on the high gray value gradient ridges. So "border_on_ridges" closer to 0.0 is better, "avg_dist2ridges" closer to 0.0 is better, and "max_dist2ridges" closer to 0.0 is better.

\section{Results and discussion}

We have applied the variants for extraction of the thresholds to 81 clinical volume datasets ( 24 of them with a resolution of $256 \times 256 \times 256$ voxels, the rest $128 \times 128 \times 128$ voxels), acquired with the 3D Integris system [12]. The voxel size varies between 0.2 and 1.2 millimeter.

The median $M D$, mean $M N$ and maximum $M X$ of the three characteristic numbers (Sec. 2.4) of the 81 clinical volume datasets are given in Table 1.

The following conclusions can be drawn from the results and the experience gained:

1. Since the GR threshold is derived from the gray values of the gradient ridge voxels, the GR threshold gives the best (i.e. lowest) results. But, since the GR threshold is a global threshold (as all thresholds used for segmentation of the 81 clinical volume datasets) and since the gray values of the gradient ridge voxels vary considerably (Fig. 5 of [9]), the median, mean and maximum are 
still not close to zero. So, a better segmentation is probably only possible by local thresholds.

2. The OT threshold gives compared to the GMM, KI and RF thresholds good results except for the maximum (MX) of the characteristic numbers "avg_dist2ridges" and "max_dist2ridges". However, it was not possible to extract an OT threshold for three of the 81 clinical volume datasets.

3. The KI threshold and the RF threshold give more or less the same results. However, it was not possible to extract an KI threshold for one of the 81 clinical volume datasets.

4. The GMM threshold gives the worst results, especially for the mean (MN) and the maximum (MX) of the characteristic numbers "avg_dist2ridges" and "maxdist2ridges". This indicates that a twofold Gaussian mixture model is not always a correct assumption for the distribution of the gray values.

\section{References}

1. Moret J, Kemkers R, de Beek JO, et al. 3D rotational angiography: Clinical value in endovascular treatment. Medicamundi. 1998;42(3):8-14.

2. Kittler J, Illingworth J. Minimum error thresholding. Pattern Recognit. 1986;19(1):41-47.

3. Gan R, Wong WCK, Chung ACS. Statistical cerebrovascular segmentation in three-dimensional rotational angiography based on maximum intensity projections. Med Phys. 2005;32(9):3017-3028.

4. Bruijns J. The expectation-maximization algorithm for a twofold Gaussian mixture model applied to the histogram of the observations. Proc CAR. 2008; p. S392-S393.

5. Bilmes J. A Gentle Tutorial on the EM Algorithm and its Application to Parameter Estimation for Gaussian Mixture and Hidden Markov Models. Berkeley, CA, USA: University of Berkeley; 1997. ICSI-TR-97-021.

6. Dempster AP, Laird NM, Rubin DB. Maximum likelihood from incomplete data via the EM algorithm. J Royal Statist Soc B. 1977;39(1):1-38.

7. Otsu N. A threshold selection method from gray level histograms. IEEE Trans Sys Man Cybern. 1979;9(1):62-66.

8. Bruijns J. Verification of the self-adjusting probe: Shape extraction from cerebral vasculature. Proc VMV. 2003; p. 159-166.

9. Bruijns J. Segmentation of vessel voxel structures using gradient ridges. Proc VMV. 2003; p. 391-398.

10. Canny J. A Computational approach to edge detection. IEEE Trans Pattern Anal Machine Intell. 1986;8(6):679-698.

11. Borgefors G. Distance transformations in arbitrary dimensions. Computer Vis Graph Image Process. 1984;27(3):321-345.

12. Philips-Medical-Systems-Nederland. INTEGRIS 3D-RA. Instructions for use. Release 2.2. Best, The Netherlands: Philips Medical Systems Nederland; 2001. 9896 00132943. 